\title{
Optimal Resource Allocation for Transmitting Network Information and Data in Wireless Networks
}

\author{
Jun Hong and Victor O.K. Li \\ Department of Electrical and Electronic Engineering \\ The University of Hong Kong, Pokfulam Road, Hong Kong, China \\ Email: \{hongjun,vli\}@eee.hku.hk
}

\begin{abstract}
The capacity of wireless networks is greatly affected by the available information on network states, such as network topology, channel state, and traffic information. Previous research has estimated the capacity of wireless networks by assuming that each node in the network can obtain precise network information. However, in reality, precise network information may not be readily available, and it may require a large amount of bandwidth resource to maintain accurate network information, thus reducing the net data rate. In this paper, we study the tradeoff between network performance improvement and the communication overhead of transmitting network information. To do so, we first determine the resource required to transmit network information reliably. A channel model is presented for the transmission of network information packets, and the network protocols are modeled as coding schemes. An informationtheoretic method is used to obtain the lower bound on the resource required to maintain accurate network information. We use the result to find the optimal allocation of resource between network information transmission and data transmission so as to maximize the net data rate. The model can also be used to derive the upper bound on wireless network performance for topology-transparent algorithms.
\end{abstract}

\section{INTRODUCTION}

As wireless networks evolve, there is a trend toward decentralized and self-organizing networks, in which network information, such as network topology, channel state and traffic information, may be transmitted to assist network transmission decisions. Previous research on the performance of wireless networks has provided an upper bound on the achievable performance by assuming that precise network information is available. Gupta and Kumar first determine the capacity of wireless networks under some idealized assumptions [1]. Franceschetti et al. close the gap between the capacity upper and lower bounds in Gupta and Kumar's original results under the physical model [2]. Zhang and Hou extend this work to networks with unlimited bandwidth [3]. Some researchers analyze the impact of interference on multi-hop wireless network performance. Jain et al. use a conflict graph to model the wireless interference and compute upper and lower bounds on the network throughput [4]. Kodialam and Nandagopal analyze the effect of interference on the achievable data rate in wireless networks [5]. Kolar and Abu-Ghazaleh study the performance of globally aware routing which is cognizant of the wireless interference [6]. The scheduling effects on wireless network performance have also been studied. Garetto et al. use a Markovian model to estimate the effects of scheduling on the throughput of CSMA channels [7]. Kolar and Abu-Ghazaleh evaluate the scheduling interactions among several given links and analyze the scheduling effects on network capacity [8].

We observe that wireless network performance depends not only on the wireless interference or the scheduling algorithm, but also on the available network information. However, previous research determines the capacity of wireless networks by assuming the existence of an omniscient, which can obtain precise network information and perform perfect scheduling. In reality, available network information is mostly imprecise and incomplete. Furthermore, in wireless networks, obtaining network information may require large amount of bandwidth resource. So, the objective of this paper is to study the tradeoff between network performance improvement and the communication overhead of transmitting network information. In our previous work [9], we have studied the minimum quantity of network information required to achieve a given level of network performance, where the network information is treated as an information source, and the minimum information is found by minimizing the code letters required to encode the source. But how much resource should be allocated for transmitting those code letters? Intuitively, when the capacity of the network is small, it may take a large part of the network bandwidth resource to transmit network information reliably. In such cases, the optimal network performance may be achieved by allowing erroneous information transmissions. So, in this paper, we will answer the following two questions:

1) How much resource should be allocated for transmitting network information reliably in an environment with wireless interference?

2) What is the optimal resource allocation for transmitting network information and data so as to maximize the network performance.

In this paper, a packet transmission is modeled as an input character of a noisy channel, where all the wireless interference acts like the noise of the channel. We find that any given scheduling protocol can be modeled as a coding scheme, by simply mapping each packet to a code letter. So, without searching for the optimal protocol, the lower bound 
on the transmitting rate which ensures zero probability of error can be derived by applying the channel coding theorem. The model can also be used to obtain the upper bound on the performance of topology-transparent algorithms presented in [10]-[12]. Since the optimal network performance may be achieved when there are contentions among network information transmissions, we analyze the relationship between the failure rate and the transmitting rate of the network information, and find the quantity of network resource that should be allocated for information transmissions to achieve the optimal network performance.

The main contributions of this paper are:

1) We model a packet transmission as a character going through a noisy channel. We find that, for each transmission, the input distribution of the channel determines the effect of this transmission on other nodes. By considering the relationship between the input distribution and the transition matrix of the channels, the interactions among nodes in the network are modeled accurately.

2) In this work, we study the tradeoff between network performance improvement and information collection overhead. The maximum network performance and the optimal resource allocation scheme is derived by considering the communication overhead.

3) Almost all existing work on wireless network performance makes the unrealistic assumption that there is an omniscient who has perfect information and performs perfect scheduling. Different from previous work, we derive performance upper bound without assuming such an omniscient.

The rest of the paper is organized as follows. Section II describes the network model used throughout the paper. Section III analyzes the lower bound on the resource required to transmit a given quantity of network information. Section IV studies how to allocate the resource between information transmission and data transmission so as to achieve the optimal network performance. Section V studies the upper bound on wireless network performance. Section VI concludes the paper.

\section{NETWORK MODEL}

As in [1], we scale space and suppose that $N$ nodes are uniformly located in a region of area $1 \mathrm{~m}^{2} . n_{i}$ denotes the $i$-th node, and $d_{i j}$ denotes the distance between nodes $n_{i}$ and $n_{j}$. The communication range is $R_{C}$, i.e., each node can transmit with a maximum radius $R_{C} . R_{I}(r)$ denotes the interference range when the communication range $R_{C}$ is equal to $r$. According to [13], $R_{I}(r)=\rho r$, where $\rho \approx 1.78$. Let $S_{I}(r)$ denote the interference area when the communication range is $r$, and $S_{I}(r)=\pi(\rho r)^{2}$. It is assumed that when transmitting data, the communication range of each node is set to be $r_{C}$. Since nodes may want information beyond $r_{C}$, the communication range is set to be $r_{D}$ when transmitting network information, and usually $r_{D}>r_{C}$.

We introduce an idle character, denoted _. For each senderreceiver pair, when the sender is idle, we regard it as equivalent to sending an idle character _. Obviously, the "transmission" of the idle character will not cause any wireless interference or have any impact on the performance of other nodes. Note that except for the idle character, any other message will cause wireless interference. If a transmission of a message fails due to wireless interference, the receiver is regarded as having received a character $f$, which indicates noise.

We use the protocol interference model [1] to define the conditions for a successful wireless transmission.

Protocol Interference Model: the transmission from node $n_{i}$ to $n_{j}$ is successful if

1) $d_{i j} \leq R_{C}$

2) any node $n_{k}$, such that $d_{k j} \leq R_{I}$, is not transmitting.

When transmitting network information, each node uses a fixed power which is large enough to broadcast network information to all the nodes within $r_{D}$. Therefore, for each receiver, the information is received successfully if none of the nodes within its interference range, which is equal to $\rho r_{D}$, is transmitting non-idle characters.

\section{RESOURCE BOUND FOR NETWORK INFORMATION}

In this section, we analyze the minimum network resource required for transmitting network information reliably in an environment with wireless interference.

If there is no wireless interference, the network information can be transmitted accurately. Otherwise, a packet collision happens and the transmission of the network information fails. So, for each link, two discrete channels shown in Figure 1 can be used to model the transmission of the network information packets, each of which is mapped to an input letter of the channel. For the $i$-th link, suppose the input $X_{i}$ takes values in $\left\{w_{1}, w_{2}, \cdots, w_{n},{ }_{-}\right\}$, and the output $Y_{i}$ takes values in $\left\{w_{1}, w_{2}, \cdots, w_{n},-f\right\}$, where is the idle character, and $f$ is the noise character. As shown in Figure 2, we assume that, as in a time division multiple access system, the packets are synchronized and with the same length.

We use a variable $s_{i}$ to indicate whether there is wireless interference affecting transmission on the $i$-th link. When $s_{i}$ is 0 , which means there is no wireless interference, the input characters can be received accurately. When $s_{i}$ is 1 , the transmission fails, and the output $Y$ will receive the noise character $f$ with probability 1 . Suppose the distribution of $s_{i}$ is given by

$$
P\left(s_{i}=0\right)=1-\varepsilon_{i}, \text { and } P\left(s_{i}=1\right)=\varepsilon_{i},
$$

where $0 \leq \varepsilon_{i} \leq 1$.

The probability distribution of $s_{i}$ (i.e. the value of $\varepsilon_{i}$ ) depends on the behavior of the other nodes in the network. Intuitively, when each node sends a non-idle character with higher probability, the probability of collision increases, thereby leading to a larger $\varepsilon_{i}$.

It has been proved that if neither the decoder (receiver) nor the encoder (sender) gets the information of $s_{i}$, the interference acts as noise and the capacity of the channel is the same as the one depicted in Figure 3 [14]. So, for the $i$-th link, the 

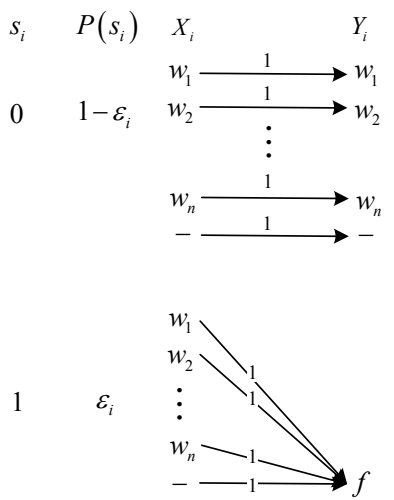

Fig. 1. Transition diagram of channel $i$ given $s_{i}$

\begin{tabular}{|c|c|c|c|c|c|}
\hline \multicolumn{4}{|c|}{ Packet transmissions } & \multirow{3}{*}{$\begin{array}{l}\cdots \\
\cdots\end{array}$} & nding code \\
\hline$W_{1}$ & W1 & W1 & W1 & & $W_{l} W_{l} W_{l} W_{l}$ \\
\hline W2 & & W2 & & & $W_{2}-W_{2}-$ \\
\hline W3 & & & W3 & $\cdots$ & $W_{3}-W_{3}$ \\
\hline
\end{tabular}

Fig. 2. Transmissions of Packets

quantity of information the receiver can obtain is

$$
I\left(X_{i} ; Y_{i}\right)=\sum_{j=1}^{n} Q_{i}\left(w_{j}\right) I\left(w_{j} ; Y\right)+Q_{i}\left({ }_{-}\right) I\left({ }_{-} ; Y\right)
$$

where $Q_{i}(x)$ is the probability that link $i$ sends a character $x$, and $\sum_{j=1}^{n} Q_{i}\left(w_{j}\right)+Q_{i}\left(_{-}\right)=1$.

Let $\varepsilon^{N}$ denote the vector $\left(\varepsilon_{1}, \cdots, \varepsilon_{N}\right)$, and $Q^{N}\left(\_\right)$denote $\left(Q_{1}\left(\_\right), \cdots, Q_{N}\left(\_\right)\right)$. Intuitively, we hope $\varepsilon^{N}$, which indicates the probability of error, to be as small as possible, so that the receiver can receive the information more accurately. However, since the links in wireless networks are not isolated, the transition matrix of each channel depends on the input distributions of the other channels. For the $i$-th channel, $\varepsilon_{i}$ will only decrease when other nodes transmit an idle character with larger probability. So, when $\varepsilon^{N}$ becomes smaller, although the noise of each channel becomes smaller, and each packet can be received accurately with larger probability, the information contained in each packet may become less, which will lead to a smaller channel capacity. An extreme case is when $\varepsilon^{N}=(0, \cdots, 0)$, then $Q^{N}\left(\_\right)=(1, \cdots, 1)$. Though all the packets can be received without error, the entropy of each packet is 0 , and the corresponding network capacity is 0 .

To find the minimum network resource required to transmit a given quantity of network information is equivalent to finding the maximum quantity of information that can be transmitted successfully through the network per unit time. So, we define our objective function as

$$
G=\max _{Q^{N}=\left(\overrightarrow{Q_{1}}, \overrightarrow{Q_{2}}, \cdots, \overrightarrow{Q_{N}}\right)} \sum_{i=1}^{N} I\left(X_{i} ; Y_{i}\right)
$$

where $\overrightarrow{Q_{i}}=\left(Q_{i}\left(w_{1}\right), \cdots, Q_{i}\left(w_{n}\right), Q_{i}\left({ }_{-}\right)\right)$is the input distribution of the $i$-th channel. The objective is to find

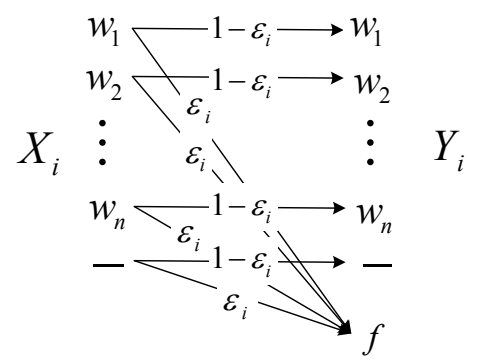

Fig. 3. Transition diagram of the channel

an optimal combination of the input distributions of all the channels, so that the network capacity can be maximized.

For a transmission on the $i$-th link, a node $n_{k}$ may interfere with the transmission if two conditions are satisfied,

1) $n_{k}$ is within the interference range of the receiver, and this occurs with probability $S_{I}\left(r_{D}\right)$.

2) $n_{k}$ is transmitting a non-idle character, and this occurs with probability is $1-Q_{k}\left({ }_{-}\right)$.

So, the probability that this transmission has a collision with $n_{k}$ is $S_{I}\left(r_{D}\right)\left(1-Q_{k}\left({ }_{-}\right)\right)$. Since there are $N$ nodes in the network, and the transmission will be successful if none of the nodes collides with it, the probability of a successful transmission is given by

$$
1-\varepsilon_{i}=\prod_{k=1, k \neq i}^{N}\left(1-S_{I}\left(r_{D}\right)\left(1-Q_{k}\left(\_\right)\right)\right)
$$

Suppose $\left(Q_{i}\left(w_{1}\right), \cdots, Q_{i}\left(w_{n}\right)\right)$ is denoted by $\overrightarrow{Q_{i}}\left(w^{n}\right)$, and $\left(\overrightarrow{Q_{1}}\left(w^{n}\right), \cdots, \overrightarrow{Q_{N}}\left(w^{n}\right)\right)$ is denoted by $Q^{N}\left(w^{n}\right)$. From Equation (1), we can see that the value of $\varepsilon^{N}$ depends only on $Q^{N}\left({ }_{-}\right)$, namely, when $Q^{N}\left({ }_{-}\right)$is fixed, $I\left(X_{i} ; Y_{i}\right)$ depends only on $\overrightarrow{Q_{i}}\left(w^{n}\right)$, and $\overrightarrow{Q_{i}}\left(w^{n}\right)$ will not affect $I\left(X_{j} ; Y_{j}\right)(i \neq j)$. So, the network capacity can be written as

$$
G=\max _{\left.Q^{N}()^{-}\right)} \sum_{i=1}^{N} \max _{\overrightarrow{Q_{i}}\left(w^{n}\right)} I\left(X_{i} ; Y_{i}\right)
$$

As shown in Figure 3, since the transition matrix of $w_{1}, w_{2}, \cdots, w_{n}$ is symmetric, the mutual information is maximized when the inputs $w_{1}, w_{2}, \cdots, w_{n}$ are with equal probability [15], i.e., $Q_{i}\left(w_{1}\right)=Q_{i}\left(w_{2}\right)=\cdots=Q_{i}\left(w_{n}\right)=\alpha_{i}$. Note that with efficient source coding, the input characters (packets) should have the same probability. We assume that the source coding is efficient, and the condition that $Q\left(w_{1}\right)=$ $\cdots=Q\left(w_{n}\right)$ can always be satisfied. So,

$$
\begin{aligned}
\underset{Q_{i}\left(w^{n}\right)}{\max } I & \left(X_{i} ; Y_{i}\right)=\left(1-\varepsilon_{i}\right)\left(n \alpha_{i} \log n\right)- \\
& \left(1-\varepsilon_{i}\right)\left(n \alpha_{i} \log n \alpha_{i}+\left(1-n \alpha_{i}\right) \log \left(1-n \alpha_{i}\right)\right)
\end{aligned}
$$

Note that $n \alpha_{i} \log n \alpha_{i}+\left(1-n \alpha_{i}\right) \log \left(1-n \alpha_{i}\right) \leq 1$ bit. So, when $n$ is large, which is exactly the case in most practical systems (because $n=2^{l}$, where $l$ is the length of each packet), we have

$$
\max _{\overrightarrow{Q_{i}}\left(w^{n}\right)} I\left(X_{i} ; Y_{i}\right) \approx\left(1-\varepsilon_{i}\right) n \alpha_{i} \log n
$$


Then the network capacity is given by

$$
G=n \log n \max _{\alpha^{N}} \sum_{i=1}^{N} \alpha_{i} \prod_{k=1, k \neq i}^{N}\left(1-S_{I}\left(r_{D}\right) n \alpha_{k}\right)
$$

where $\alpha^{N}=\left(\alpha_{1}, \cdots, \alpha_{N}\right)$.

The optimization problem in Equation (4) can be solved by the Lagrange Multiplier Method. The solution is given by

$$
\left\{\begin{array}{l}
\forall i, \quad n \alpha_{i}=0 \text { or } 1 \\
\sum_{i=1}^{N} n \alpha_{i}=m
\end{array}\right.
$$

where

$$
m= \begin{cases}N & \text { if } \beta \geq N \\ \lceil\beta\rceil & \text { if } \beta<N \text { and } \frac{\lfloor\beta\rfloor}{\lceil\beta\rceil}<1-S_{I}\left(r_{D}\right) \\ \lfloor\beta\rfloor & \text { otherwise. }\end{cases}
$$

and $\beta=-\frac{1}{\ln \left(1-S_{I}\left(r_{D}\right)\right)}$.

The optimal solution is that, at any time, the number of active nodes, i.e., nodes transmitting non-idle characters, is $m$. The corresponding network capacity is given by

$$
G=m\left(1-S_{I}\left(r_{D}\right)\right)^{m-1} \log n .
$$

Since there are $N$ nodes in the network, the capacity of each channel is

$$
C=\frac{G}{N}
$$

Suppose $R$ is the data rate in bits per packet, and $R=\frac{\log n}{L}$, where $L$ is the number of time slots for each packet. According to the Channel Coding Theorem, we have $R<C$, and

$$
L>\frac{\log n}{C}=\frac{N}{m\left(1-S_{I}\left(r_{D}\right)\right)^{m-1}}
$$

So, to transmit a packet successfully, at least $\frac{N}{m\left(1-S_{I}\left(r_{D}\right)\right)^{m-1}}$ time slots are required.

Note that the result is based on the assumption that the nodes in the network are uniformly distributed. Some may argue that the result cannot be applied to a given fixed topology. In fact, wireless networks are typically mobile, and a fixed network with precise topology information available is not our focus. Our result can be interpreted as the time weighted average performance of a mobile network where the nodes are uniformly distributed at each snapshot. When there is no precise topology information, the best the nodes can do is to follow Equation (5), so that the expected network throughput over all possible topologies is maximized.

\section{Analysis of Resource Allocation}

In Section III, we determine the amount of resource required to transmit the network information without error. However, insisting on a zero failure rate of network information transmission will consume valuable network resource. The consumed resource may be more than that gained due to using this more accurate information. In this section, we study the optimal resource allocation between information transmission and data transmission.
According to the result in [15], an error probability per source digit of $\delta$ can be achieved through appropriate coding if the channel capacity $C^{\prime}$ (in bits per source symbol) satisfies

$$
C^{\prime}>A-h(\delta)-\delta \log (K-1)
$$

where $A$ is the entropy of the source in bits, $K$ is the alphabet size of the source, and $h(\delta)=-\delta \log \delta-(1-\delta) \log (1-\delta)$.

Suppose the length of the code for each source letter is $\lambda$, i.e., the number of channel uses per source symbol is $\lambda$, and the channel capacity per channel use is $C_{0}$. Then $C^{\prime}=\lambda C_{0}$. So, to achieve an error probability of $\delta$, the minimum length of the code for each source symbol is

$$
\lambda_{\text {min }}=\frac{A-h(\delta)-\delta \log (K-1)}{C_{0}}
$$

Now we will study how much resource should be allocated for network information transmission by analyzing the Multiple Access Collision Avoidance (MACA) [16] protocol which uses Request-To-Send (RTS) and Clear-To-Send (CTS) to prevent collisions. If a node wants to send a packet, it first sends an RTS signal. If the receiver gets the RTS signal and it is available, it returns a CTS signal. The basic idea of MACA is that any node that overhears a CTS signal has to defer its own transmissions for the length of the data transmission so as to avoid collisions. However, collisions may still occur when the CTS signal is not received by some nodes which happen to have transmissions.

Suppose the nodes in the network are homogeneous. Consider a data transmission between a sender $n_{i}$ and a receiver $n_{j}$. According to MACA, prompted by an RTS from $n_{i}, n_{j}$ broadcasts a CTS signal. Let $p_{e}$ be the probability that the CTS signal is not received by a node which may interfere with the transmission. Let $p_{t}$ be the probability that a neighboring node of $n_{j}$ transmits conditional on the fact that it does not receive the CTS signal sent by $n_{j}$. Then for each node, the probability that it has a conflict with the transmission from $n_{i}$ to $n_{j}$ is $S_{I}\left(r_{C}\right) p_{e} p_{t}$, where $r_{C}$ is the communication range when transmitting data. As in [17], we assume that the probability each node collides with the packet is independent, then the probability of a successful transmission is

$$
p_{s}=\left(1-S_{I}\left(r_{C}\right) p_{e} p_{t}\right)^{N-2}
$$

Suppose each CTS packet is $\log n$ bits, and the channel capacity in bits per packet is $C$. According to Equation (8), to achieve an error probability of $p_{e}$, the number of bits required for transmitting a CTS signal is

$$
u_{c}=\log n \times \frac{\log n-h\left(p_{e}\right)-p_{e} \log (n-1)}{C}
$$

where $C$ is given by Equation (7).

Suppose the average packet payload size is $u$. The net data rate $r_{N}$ is defined as the average amount of payload information transmitted successfully per channel use, then

$$
E\left(r_{N}\right)=\frac{u p_{s}}{u+u_{c}}
$$


Substituting Equations (9) and (10) in (11), we have

$$
E\left(r_{N}\right) \approx \frac{u\left(1-S_{I}\left(r_{C}\right) p_{t} p_{e}\right)^{N-2}}{u+\frac{(\log n)^{2}}{C}\left(1-p_{e}\right)}
$$

The optimal value of $p_{e}$ is given by,

$$
p_{e}^{*}= \begin{cases}0 & \text { if } \frac{u C}{u C+(\log n)^{2}} \geq \frac{\left(1-S_{I}\left(r_{C}\right) p_{t}\right)^{N-2}}{u} \\ 1 & \text { otherwise }\end{cases}
$$

Therefore, to achieve the optimal net data rate, when $\frac{u C}{u C+(\log n)^{2}} \geq \frac{\left(1-S_{I}\left(r_{C}\right) p_{t}\right)^{N-2}}{u}$, the receiver should not transmit any CTS packet; otherwise, $\frac{(\log n)^{2}}{C}$ bits are required for transmitting the CTS signal.

\section{Network CAPACITY Without NeTwORK INFORMATION}

Previous research has determined the performance bounds for wireless networks by assuming that precise network information can be derived. In reality, however, available network information is mostly imprecise and incomplete. Furthermore, in wireless networks, obtaining network information may require large amount of bandwidth resource. So, many topologytransparent algorithms have been proposed to maximize the network performance [10]-[12]. The network model defined in Section II may be used to analyze topology-transparent algorithms. It is assumed that all nodes always have packets waiting to send, and each node chooses one of its neighbors (if it has any) randomly to start a transmission. Suppose the network runs the TDMA protocol, and the rate of the wireless channel is $W$ bits/sec. The network throughput $\mu$ is defined as the quantity of information (in bits) transmitted in the network per second. Let $\mu_{i}$ be the quantity of information transmitted by $n_{i}$ per second, then we have

$$
E(\mu)=E\left(\sum_{i=1}^{N} \mu_{i}\right)=\sum_{i=1}^{N} E\left(\mu_{i}\right)
$$

For each node, if it does not have any neighbors, it can not perform any transmission. Let $p_{c}$ be the probability of having neighbors, and $p_{c}=1-\left(1-\pi r_{C}^{2}\right)^{N-1}$. According to the result in Section III, for each link, the average number of bits transmitted successfully by sending a packet is $C$. Then

$$
E\left(\mu_{i}\right)=p_{c} W C / l
$$

where $l$ is the length of each packet, and

$$
E(\mu)=p_{c} W G / l=p_{c} W m\left(1-S_{I}\left(r_{C}\right)\right)^{m-1}
$$

\section{CONCLUSIONS}

In this paper, we have presented a channel model and an information-theoretic methodology for computing bounds on the resource required to transmit network information reliably in wireless networks. By noting that the optimal network performance may be achieved when erroneous network information transmission is allowed, we study the relationship between the failure rate and the transmitting rate of the network information, and find the optimal allocation of network resource between information and data transmissions.

Using the proposed channel model, we have also analyzed the performance of wireless networks where no information is exchanged, such as in a topology-transparent algorithm. The results obtained are more realistic than existing work which assumes perfect information is available.

\section{ACKNOWLEDGEMENT}

This research is supported in part by the University of Hong Kong Strategic Research Theme of Information Technology.

\section{REFERENCES}

[1] P. Gupta and P. Kumar, "The capacity of wireless networks," IEEE Transactions on Information Theory, vol. 46, no. 2, pp. 388-404, 2000.

[2] M. Franceschetti, O. Dousse, D. Tse, and P. Thiran, "Closing the gap in the capacity of wireless networks via percolation theory," IEEE Transactions on Information Theory, vol. 53, no. 3, pp. 1009-1018, 2007.

[3] H. Zhang and J. Hou, "Capacity of wireless ad-hoc networks under ultra wide band with power constraint," in Proceedings 24th IEEE International Conference on Computer Communications (INFOCOM 2005, vol. 1, 2005, pp. 455-465.

[4] K. Jain, J. Padhye, V. N. Padmanabhan, and L. Qiu, "Impact of interference on multi-hop wireless network performance," in Proceedings 9th annual international conference on mobile computing and networking (MobiCom '03), 2003, pp. 66-80.

[5] M. Kodialam and T. Nandagopal, "The effect of interference on the capacity of multi-hop wireless networks," in Proceedings International Symposium on Information Theory (ISIT 2004), 2004, p. 472.

[6] V. Kolar and N. Abu-Ghazaleh, "A multi-commodity flow approach for globally aware routing in multi-hop wireless networks," in Proceedings 4th Annual IEEE International Conference on Pervasive Computing and Communications (PerCom 2006), 2006.

[7] M. Garetto, T. Salonidis, and E. W. Knightly, "Modeling per-flow throughput and capturing starvation in CSMA multi-hop wireless networks," in Proceedings 25th IEEE International Conference on Computer Communications (INFOCOM 2006), 2006, pp. 1-13.

[8] V. Kolar and N. Abu-Ghazaleh, "The effect of scheduling on link capacity in multi-hop wireless networks," Arxiv preprint cs.NI/0608077, 2006.

[9] J. Hong and V. O. K. Li, "Impact of Information on Network Performance - An Information-Theoretic Perspective," in IEEE Global Telecommunications Conference, 2009. GLOBECOM'09.

[10] I. Chlamtac and A. Farago, "Making transmission schedules immune to topology changes in multi-hop packet radio networks," IEEE/ACM Transaction on Networking, vol. 2, no. 1, pp. 23-29, 1994.

[11] J.-H. Ju and V. O. K. Li, "An optimal topology-transparent scheduling method in multihop packet radio networks," IEEE/ACM Transaction on networking, vol. 6, no. 3, pp. 298-306, 1998.

[12] — "TDMA scheduling design of multihop packet radio networks based on latin squares," IEEE Journal on Selected Areas in Communications, vol. 17, no. 8, pp. 1345-1352, 1999.

[13] K. Xu, M. Gerla, and S. Bae, "How effective is the IEEE 802.11 RTS/CTS handshake in ad hoc networks," in Proceedings IEEE Global Telecommunications Conference, 2002. GLOBECOM'02, vol. 1, 2002.

[14] C. Heegard and A. Gamal, "On the capacity of computer memory with defects," IEEE Transactions on Information Theory, vol. 29, no. 5, pp. 731-739, 1983.

[15] R. G. Gallager, Information Theory and Reliable Communication. New York, Wiley, 1968.

[16] P. Karn, "Maca - a new channel access method for packet radio," ARRL/CRRL Amateur Radio 9th Computer Networking Conference, pp. 134-140, Sep. 1990.

[17] G. Bianchi, "Performance analysis of the IEEE 802. 11 distributed coordination function," IEEE Journal on selected areas in communications, vol. 18 , no. 3 , pp. $535-547,2000$. 